\title{
Sales Forecasting System for Chemicals Supplying Enterprises
}

\author{
Ma. Del Rocio Castillo E. , Ma. Magdalena Chain Palavicini ${ }^{1}$, Roberto Del Rio Soto ${ }^{1} \&$ M. Javier Cruz Gómez ${ }^{2}$ \\ ${ }^{1}$ Facultad de Contaduría y Administración, Universidad Nacional Autónoma de México (UNAM), Coyoacán, México \\ ${ }^{2}$ Facultad de Química, Universidad Nacional Autónoma de México (UNAM), Coyoacán, México \\ Correspondence: M. Javier Cruz Gómez, Facultad de Química, Universidad Nacional Autónoma de México (UNAM), \\ Coyoacán 04510, México. Tel: 52-55-5622-5359. E-mail: mjcg@unam.mx
}

Received: March 12, 2015

doi:10.5430/ijba.v6n3p39
Accepted: March 30, 2015

Online Published: April 21, 2015

URL: http://dx.doi.org/10.5430/ijba.v6n3p39

\begin{abstract}
In Mexico, the chemicals supplying enterprises have a hard job trying to make its operations planning. The difficulty is created by a combination of handling a large number of products and changeable consumption patterns of each customer. To simplify the operation planning task of those enterprises, a simplified system was envisaged and programmed. This free access and friendly software gives more accurate sales forecasts than the currently obtained by the chemicals suppliers. The developed software is written in PHP language (for web development dynamic content) and HTML (for making Web pages) and by the use of XAMPP software (Apache server module), runs on different operating systems. This program applies five quantitative sales forecasting techniques for each marketable product, with continuous error assessment, in order to select case by case, the monthly more accurate prediction method. The proposed system has been already applied in two companies; the first one used simple moving average, as a quantitative technique to predict its sales, and expert opinion as qualitative technique; the second company used trend projection as quantitative, and Delphi Method as qualitative techniques. Both companies consider that a suitable sales prediction is the starting point for good operations planning. For each product it is required to use a different technique, because the behavior of historical sales in each case is different. The proposed system allows identifying the lowest mistake technique to apply in the following sales monthly forecast, which is necessary to properly plan the operations in the companies under study. Based on the sales data of the last three years it is possible to make a yearly sales forecast, but each month, the monthly forecast should be updated with the last month data.
\end{abstract}

Keywords: sales forecast, sales software, forecasting techniques, prediction algorithm, minimum error

\section{Introduction and Background}

Effective planning, in short and long term, depends on demand forecasts for the company products (Heizer, J. (2009)). Good forecasts are crucial to all business aspects: Demand forecast is the only useful data until the actual demand is known. Therefore, demand forecasts guide decisions in many areas: human resources, capacity management and supply chain.

Forecasting is fundamental in any planning effort (Chase, R. (2014)). In the short term, forecast is needed to predict the material, products, services or other resources to respond to changes in demand. Forecasts allows to adjust schedules and vary labor and materials. In the long run, it is required to predict the basis for strategic changes, such as the development of new markets, new products or services and expand or build new facilities.

Sales and operations planning is a process that helps to provide better customer service, handling of lower inventories, offer shorter delivery time to customers, stabilize production rates and facilitate management business. It does not matter that, owing to changing markets, the chemicals supplying enterprises have troubles with operations scheduling and inventories management; they are a fundamental component in the national economy.

The chemicals supplying enterprises demands are highly variable in volume and presentation; their customers vary from a small hardware store, up to an auto assembler factory, passing through: pharmaceutical, chemical, petrochemical and polymer companies, paints and varnishes manufacturers, etc. In some companies, they handled up to 500 different materials and have up to 1000 clients; all of this complicates their operations planning.

Because of the above chemicals supplying enterprises problems, a system to forecast their sales with the best possible accuracy is proposed. The system, (algorithm and software) for the yearly or monthly sales prediction, calculates a 
possible sales number for each product using five techniques, evaluates the error of each of them and selects the lowest error technique; from which the sales forecast for each product is proposed. The goal is to make effective plans in order to properly satisfy the customer demands.

According to Thompson, (2014) the key to a most efficient Supply Chain is forecast, which very much depends on people, process, and technology. In order to gain participation from Sales, we need to

1) Educate Sales people so that they understand why forecasting is essential,

2) Developing a well-structured forecasting process,

3) Clearly define roles and responsibilities, and

4) Make access to systems easy to use to facilitate their participation.

\section{Forecasting Techniques}

\subsection{Forecasting Definition}

Forecasting, according to Everett et al. (2000), is to use past data to determine future events. Forecasts are often used to predict consumer demand for products or services, although they can predict a wide range of future events that could potentially influence the success way. Forecasting is the art and science of predicting future events. It may involve the management of historical data to project future, through some kind of mathematical model. It can be a subjective or intuitive prediction of future, or a combination of both, i.e., a mathematical model adjusted for the judgment of an administrator.

Forecasting, according to Corres, G. (2009), involves the estimation and analysis of future demand for a particular product, component or service, through different forecasting techniques. The forecast of future demand is central to any operations planning.

At the organizational level, the sales forecast is an essential input for any decision in many functional areas: sales, production, purchasing, finance and accounting. The forecasts are also needed in the distribution and supplying plans. The importance of a sales forecast with a manageable error margin is critical to the efficient management of inventories. This was in large part recognized by several authors (Chu \& Zhang, 2003).

\subsection{Basic Principles of Forecasting}

Anderson et al. (2011), give the next three basic principles for forecasting:

- $\quad$ Forecasts are not perfect, have an error margin.

- Most of the forecast techniques assume the existence of some stability in the system.

- $\quad$ Forecasts on aggregate products are more stable.

\subsection{Horizons of the Forecast}

Usually, a forecast is classified by the covered future time horizon. The time horizon is classified into three categories: 1. Short-Term Forecast: It has an extension of time up to one year, but usually less than three months. It is used to plan purchasing, scheduling work, determining levels of labor, assign work, and decide production levels.

2. Medium-term Forecast: Frequently a medium-term forecast, or intermediate term, has an area of between three months and three years. It is used to plan sales, production, budget and cash flow as well as to analyze different operating plans.

3. Long-term Forecast. Usually its extension is 3 years or more. These forecasts are used to plan the manufacture of new products, capital expenditures, location or expansion of facilities, and research and development.

The medium and long term forecasts are distinguished from short-term ones by three characteristics:

First, medium and long-term forecasts handle general aspects and support to administrative decisions concerning the products, plants and processes planning. The implementation of some decisions about facilities, may take 5-8 years since its conception until its termination.

Second, the short-term forecast usually employs different methodologies, than the longer ones. The mathematical techniques commonly used in short-term forecasts are: moving averages, exponential smoothing and trend extrapolation. The less quantitative methods are useful for taking decisions such as those related with the introduction of new products in the near future. 
Finally, short-term forecasts tend to be more accurate than long-term ones. Factors influencing demand change daily. Therefore, as the time horizon lengthens, the forecast accuracy decreases. So it is necessary to state that sales forecasts should be used regularly to maintain its value and integrity. After each period of sales, forecasts should be reviewed and updated.

\subsection{Forecasting}

By taking decisions, according to Taha, (2004), future plans are developed. Then the data describing the decision situation should represent what will happen in the future. For example, within inventory control, decisions are based on the nature of article demand for a determined planning horizon. Also, in financial planning, it is needed to predict the pattern of cash flow over time.

Prediction Techniques (Durbin \& Koopman, 2001) are widely used in the production management and inventory systems. They are frequently applied in a variety of situations, such as quality and process control, financial planning, marketing, investment analysis and distribution planning.

Two important prediction methods are extrapolation and causal prediction (Winston, (2005)). Extrapolation methods are used to predict future values of time series from past values of a time series. In a forecasting method by extrapolation, it is assumed that the sales patterns and trends will continue in future months. These methods do not take into account what caused the previous data, they simply assume that past trends and patterns will continue in the future. Causal prediction methods are intended to predict future values of a variable (called the dependent variable) using the above information, to estimate the relationship between the dependent variable and one or more independent variables.

The generally accepted techniques, according to Henke \& Reitch, (2001), for preparing forecasts are divided into two categories: qualitative and quantitative techniques.

The qualitative techniques are expert opinion panel, Delphi method, sales force composition and market survey.

The quantitative techniques are time-series and causal techniques. The time-series techniques are simple weighted, exponential moving average, double exponential smoothing with adjustable trend, trend projection and series decomposition; while the causal techniques are simple linear regression and multiple linear regression.

The choice of method or methods depends on the costs involved, the forecasting purpose, the reliability and consistency of historical sales data, the time available to make the forecast, the type of product, the market characteristics and the availability of the necessary information and expertise of those responding to the forecast. The enterprises usual practice is to combine several forecasting techniques.

\subsubsection{Qualitative Techniques}

\subsubsection{Expert Opinion Panel}

It is based on the intuition of one or more experienced executives regarding products stable demand. Its disadvantage is that it is influenced by recent events.

\subsubsection{Delphi Method}

Experts make the global and/or product by product sales predictions. A facilitator provides the experts with the average of the sales forecast and the reasons for their judgments. The experts revise their previous estimates and make new ones. The procedure is repeated until the achievement of a reasonable consensus. It is a highly accurate forecasting method.

\subsubsection{Sales Force Composition}

A technique used to project the future demand for a good or service based on the total amount that each salesperson anticipates being able to sell in their region.

\subsubsection{Market Survey}

The study of the spending characteristics and purchasing power of the consumer

\subsubsection{Quantitative Techniques}

\subsubsection{Techniques for Time Series}

Historical data from company sales is used to discover trends in seasonal, cyclical and random or erratic type. It is an effective method to produce reasonably stable demand.

Simple moving average (SMA). This technique involves calculating the average of the $\mathbf{n}$ most recent values and uses it as a forecast for the next period. By moving averages it is possible to determine if there is a seasonal factor within the time interval under consideration. 


$$
S M A=\frac{\text { Sum of }(\mathrm{n}) \text { previous values }}{\mathrm{n}}
$$

Weighted moving average (WMA). This technique involves calculating the average of the $\mathbf{n}$ most recent values giving a different "weight" in the forecast for each of the values involved.

$$
W M A=\frac{\sum \text { Value } * \text { Weigth }}{\sum \text { Weigth }}
$$

Exponential moving average (EMA). This is a type of moving average, where the forecast for the next period is based on all previous data. The most recent data has a greater weight in the forecast. The weighting for each older datum decreases exponentially, never reaching zero.

$$
\mathrm{F}_{\mathrm{t}}=\mathrm{F}_{\mathrm{t}-1}+\alpha\left(\mathrm{Y}_{\mathrm{t}-1}-\mathrm{F}_{\mathrm{t}-1}\right)
$$

Smoothing constant $\alpha=(0,1)$

$\mathrm{F}_{\mathrm{t}-1}=$ the previous forecast

$\mathrm{F}_{\mathrm{t}}=$ forecast

$\mathrm{Y}_{\mathrm{t}-\mathrm{l}}=$ previous data

Requires initialized, for example: $F_{1}=Y_{1}$

Double Exponential smoothing with adjustable trend. This is a type of smoothing that includes a special coefficient to detect a trend behavior and a coefficient for the overall behavior of the series.

$$
\begin{gathered}
F_{t}=\left(T_{t-1}\right)+(1-\alpha)\left(F_{t-1}+T_{t-1}\right) \\
T_{t}=(1-\beta) T_{t-1}+\beta\left(F_{t}-F_{t-1}\right) \\
\text { FIT }_{t}=F_{t}+T_{t}
\end{gathered}
$$

$T_{t}=$ smoothed trend for the period $t$

$\beta=$ smoothing constant for trend (0.1)

$\alpha=$ overall smoothing constant $(0.1)$

$F_{t}=$ simple exponential smoothing for the period $t$

$F I T t=$ forecast including the tendency for the period $t$

Requires initialized, for example: $F_{I}=Y_{I}$ and $T_{I}=0$

Trend Projection is used to analyze the data sets and project a linear trend in the future. The least squares method is used to find the best trend line with time as the independent variable.

$$
\mathrm{Y}=b 0+b 1 \mathrm{X}
$$

Where:

$Y=$ the predicted value

$b 1=$ the slope of the trend line

bo $=$ intercept

$X=$ time of the forecast

Series decomposition is used to analyze the data series based on the behavior of its components. There are two basic models to describe the data of a time series: Multiplicative and additive model.

Multiplicative model

$$
\mathrm{Yi}=(\mathrm{Ti})(\mathrm{Si})(\mathrm{Ii})
$$

Additive model

$$
\mathrm{Yi}=\mathrm{Ti}+\mathrm{Si}+\mathrm{Ii}
$$

Where:

$\mathrm{Ti}$ is the linear trend rate

$\mathrm{Si}$ is the index of the component seasonal 
Ii is the index of the irregular component

\subsubsection{Causal Techniques}

Simple linear regression. This is a casual approach that seeks to relate and explain the variation in the data of an indicator, as a result of a variation in a particular cause. Applied and analyzed along with the correlation coefficient and determination coefficient to interpret the explanation.

The model is:

$$
\mathrm{Y}=\mathrm{b} 0+\mathrm{b} 1 \mathrm{X}
$$

Where:

$\mathrm{b} 0$ is the intercept

$\mathrm{b} 1$ is the slope of the linear relationship

b0, b1 are obtained by the minimum squares method.

Multiple Linear Regression. Multiple linear regression attempts to model the relationship between two or more explanatory variables and a response variable by fitting a linear equation to observed data.

\subsubsection{Sources and Type of Error in the Forecast}

The present system assumes that a proper screening and correction of the data and the data errors was already made.

The software determines the forecast techniques behavior and their errors, by the following calculations:

$$
\text { Forecast error }=\text { actual value }- \text { predicted value }
$$

QME: Quadratic mean error

MAPE: Medium absolute percentage error

MAD: mean absolute deviation

\section{Most common error measurements}

$\mathrm{QME}=\Sigma$ (forecast error $)^{2} / \mathrm{n}$

$\mathrm{MAD}=\Sigma \mid$ forecast error $\mid / \mathrm{n}$

MAPE $=[\Sigma \mid$ forecast error $\mid /$ actual value $] / \mathrm{n}$

Control signal (tracking)

Tracking $=\Sigma$ (forecast error) $/$ MAD

\section{Seven Steps in the Forecasting System}

For the present development, the seven steps proposed by Heizer, J. (2009) in order to initiate, design and implement a forecasting system, were taken on account, as follows:

I. Determine the usage of Forecast: Chemicals sales forecasts will be used by the chemicals suppliers in order to better plan their operations.

II. Select the aspects to predict: Chemicals sales forecast based on each company historical sales data.

III. Determine the time horizon of the forecast: The system will be handling the short-term forecast, with an extension of time up to one year.

IV. Select forecasting models: The models used by the present system are: Simple moving average, weighted moving average, trend projection, exponential smoothing and simple linear regression. In some cases, it is also required the use of qualitative techniques.

Besides the selection of the forecasting models, it was also selected the MAPE (mean absolute percentage error), as a programmable assessment technique for the goodness degree of the forecasting models.

V. Compile the necessary data for the Forecast: Chemicals sales historical data for three years were collected and properly grouped in order to be used by the forecasting software. A database example for company 1 historical sales data (2010-2012) is shown in the following table. In the case of the company 2, 21 products were analyzed. 
Table 1. Data product 1 and 2 of the first company during 12 months of three years

\begin{tabular}{|l|r|r|r|r|r|r|r|r|r|r|r|r|r|}
\hline & 1 & 2 & 3 & 4 & 5 & 6 & 7 & 8 & 9 & 10 & 11 & 12 \\
\hline I.1 & 85,999 & 109,149 & 161,386 & 158,440 & 145,908 & 133,005 & 252,688 & 474,882 & 374,347 & 222,749 & 309,505 & 356,227 \\
\hline II.1 & 375776.328 & 377311.784 & 514939.156 & 317637.977 & 236617.238 & 254699.476 & 405059.784 & 530239.028 & 258920.28 & 319859.776 & 360972.504 & 296114.38 \\
\hline III.1 & $310,167.04$ & $388,850.60$ & $359,335.59$ & $277,029.57$ & $293,032.09$ & $315,182.73$ & $362,608.44$ & $348,899.86$ & $246,059.14$ & $304,941.33$ & $296,499.82$ & $202,061.98$ \\
\hline & 1 & 2 & 3 & 4 & 5 & 6 & 7 & 8 & 9 & 10 & 11 & 12 \\
\hline 1.2 & 337,032 & 299,402 & $381, .836$ & 387,605 & 457,823 & 525,706 & 572,672 & 449,388 & 525,816 & 374,428 & 522,622 & 310,347 \\
\hline II.2 & 503063.68 & 414015.338 & 450512.531 & 396477.384 & 412443.725 & 250992.838 & 250407.561 & 218011.621 & 303665.456 & 179865.324 & 11910.85 & 20337.2928 \\
\hline III.2 & 159,71839 & $267,212.41$ & $186,576.23$ & $148,557.23$ & $269,495.35$ & $180,390.53$ & $97,987.50$ & $309,858.61$ & $241,382.11$ & $210,572.48$ & $220,952.94$ & $138,479.35$ \\
\hline
\end{tabular}

VI. Perform Forecast: An algorithm was made (See Figure 1.) in order to: a) Calculate the sales forecast by the five chosen quantitative techniques: simple moving average, weighted moving average, exponential smoothing, trend projection and simple linear regression, b) Draw graphics of the historical data and the five techniques calculations results, c). Calculate the MAPE error and d) Select the lowest error forecast technique.

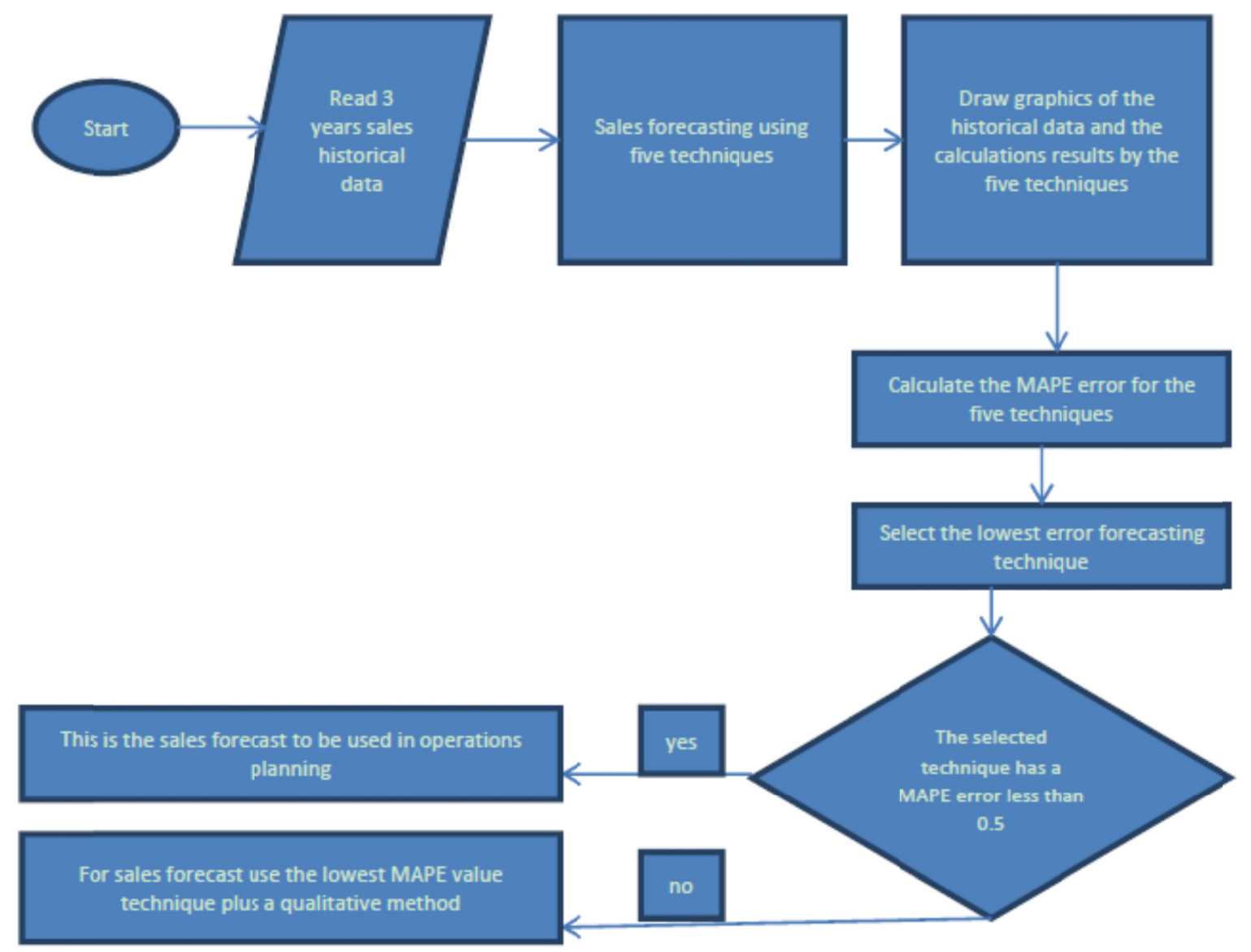

Figure 1. Algorithm used by the computer program

Once the algorithm was developed, a computer program in HTML (Hyper Text Markup Language) and PHP language (Personal Home Page Tools) was written. The apache server module of XAMPP free software is used; this module allows accommodate the program.

The program has features that allow making the calculations enumerated in the algorithm. The sales forecast is obtained from the technique with the minor error, if this has a MAPE value less than 0.5. But when the minor error technique has a MAPE value greater than 0.5, the forecast values obtained from this technique require a correction made by a qualitative method. As an example, it is mentioned that for this study, company 1 uses expert opinion and company 2 uses Delphi Method as the qualitative methods. 
A manual for the use of the computer program was also written with the following contents: system installation, system implementation considerations for archiving database and system menu. The manual and the software can be obtained from any of the authors.

VII. Validate and implement results. Program results are compared with those obtained previously in Excel to verify the proper functioning of the program developed. The test was performed both with random numbers and the data from two companies. Whoever wants to use the program should make monthly sales updates to produce the new sales forecasts.

\section{Analysis of Results}

In Figures 2, 3 and 4 are made representations, for different products, of the actual sales by the light blue lines, company sales forecast by the orange line, and the sales forecast obtained by the present system by the dark blue line. Company 1 obtained its forecasts of Figures 2 and 3 by simple moving average, and company 2 obtained its forecast of Figure 4 by trend projection. It can be seen that the system forecasts are closer to the actual sales values that the technique used by the companies.

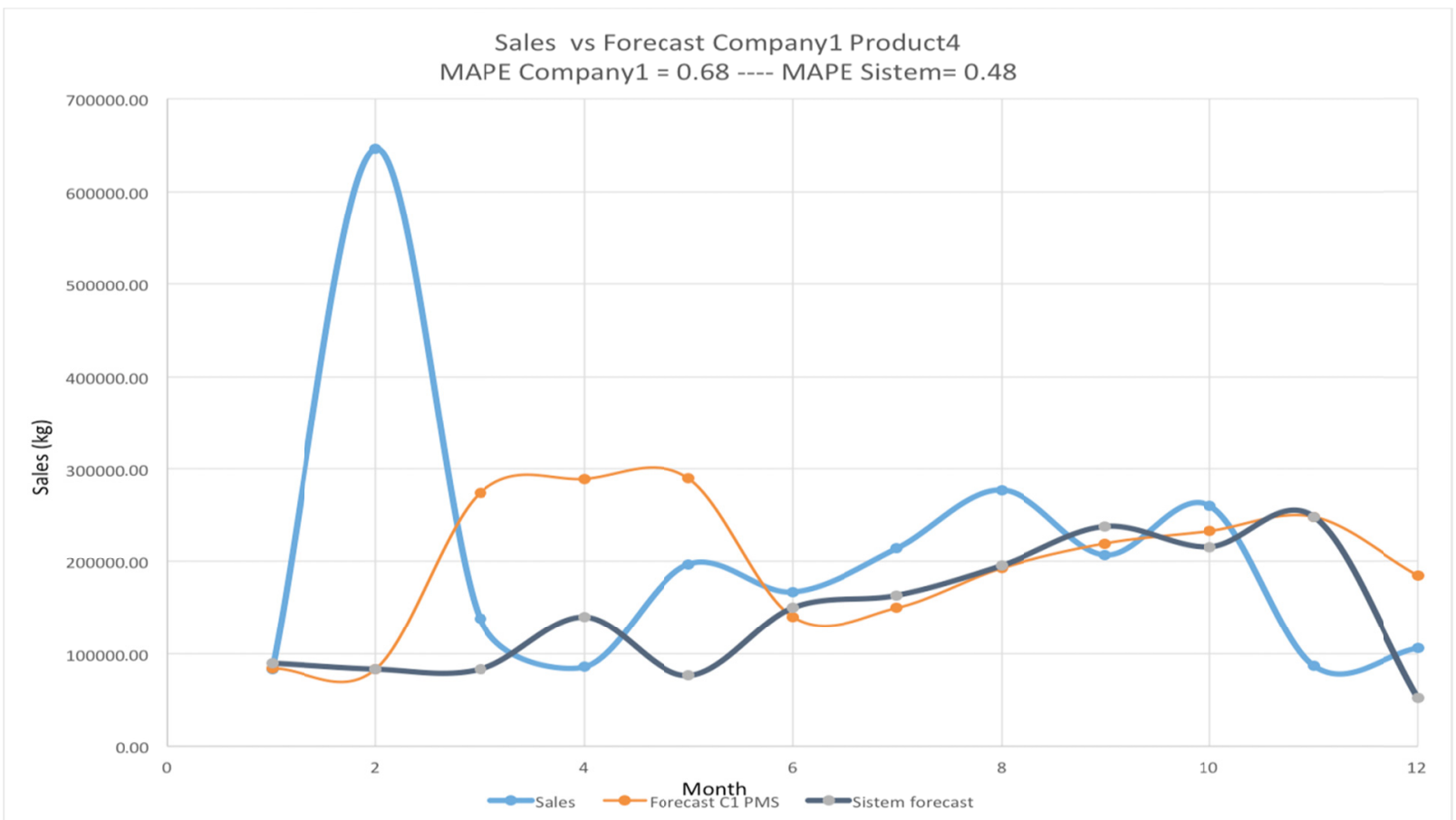

Figure 2. Comparison of the actual sales (Light blue line), with the sales forecast obtained by the simple moving technique in Company 1 (Orange line), and the values obtained by the present forecast system (Dark blue), with a

MAPE $<0.5$ 


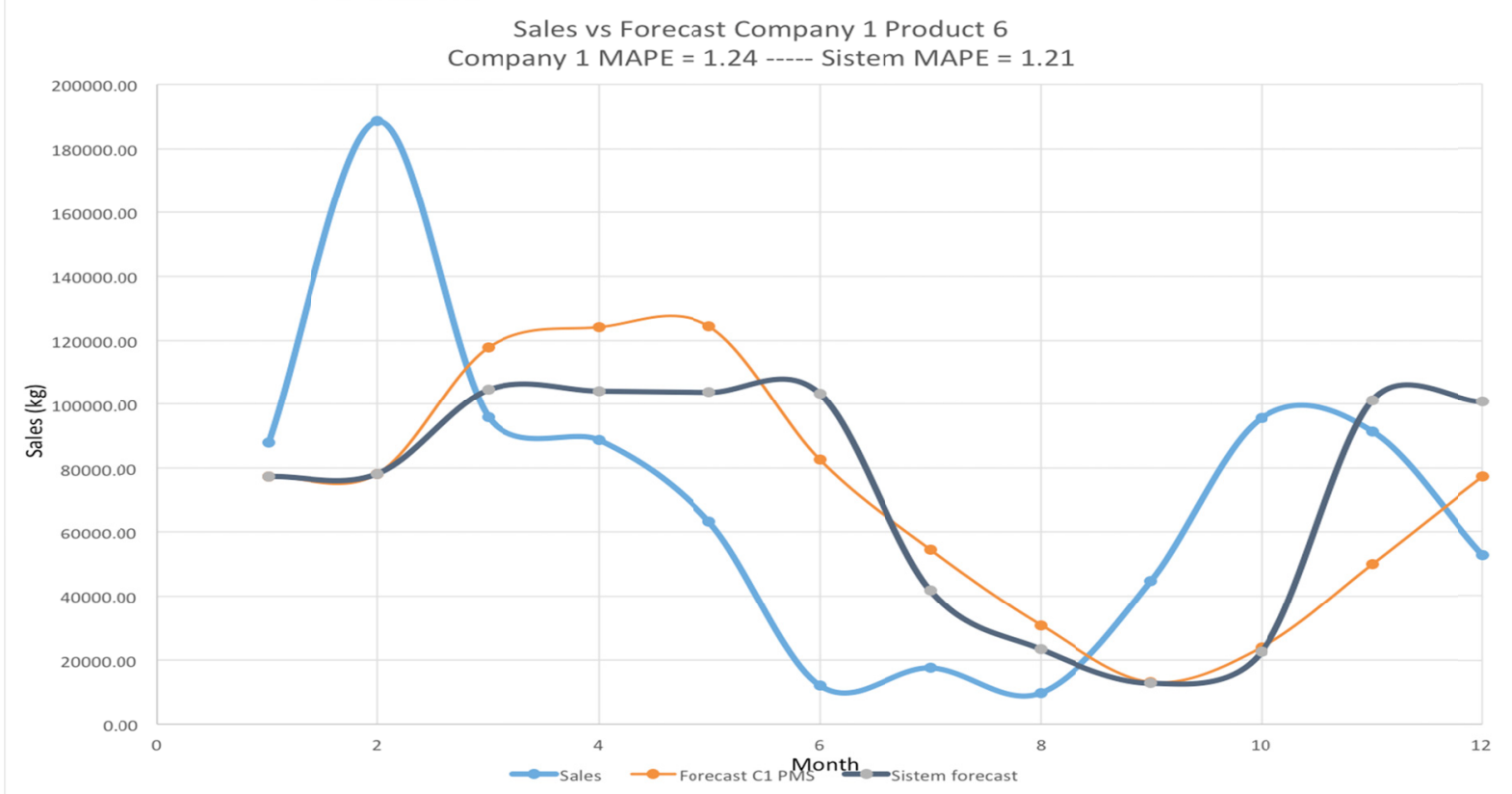

Figure 3. Comparison of the actual sales (Light blue line), with the sales forecast obtained by the simple moving technique in Company 1 (Orange line), and the values obtained by the present forecast system (Dark blue), with a MAPE $>0.5$

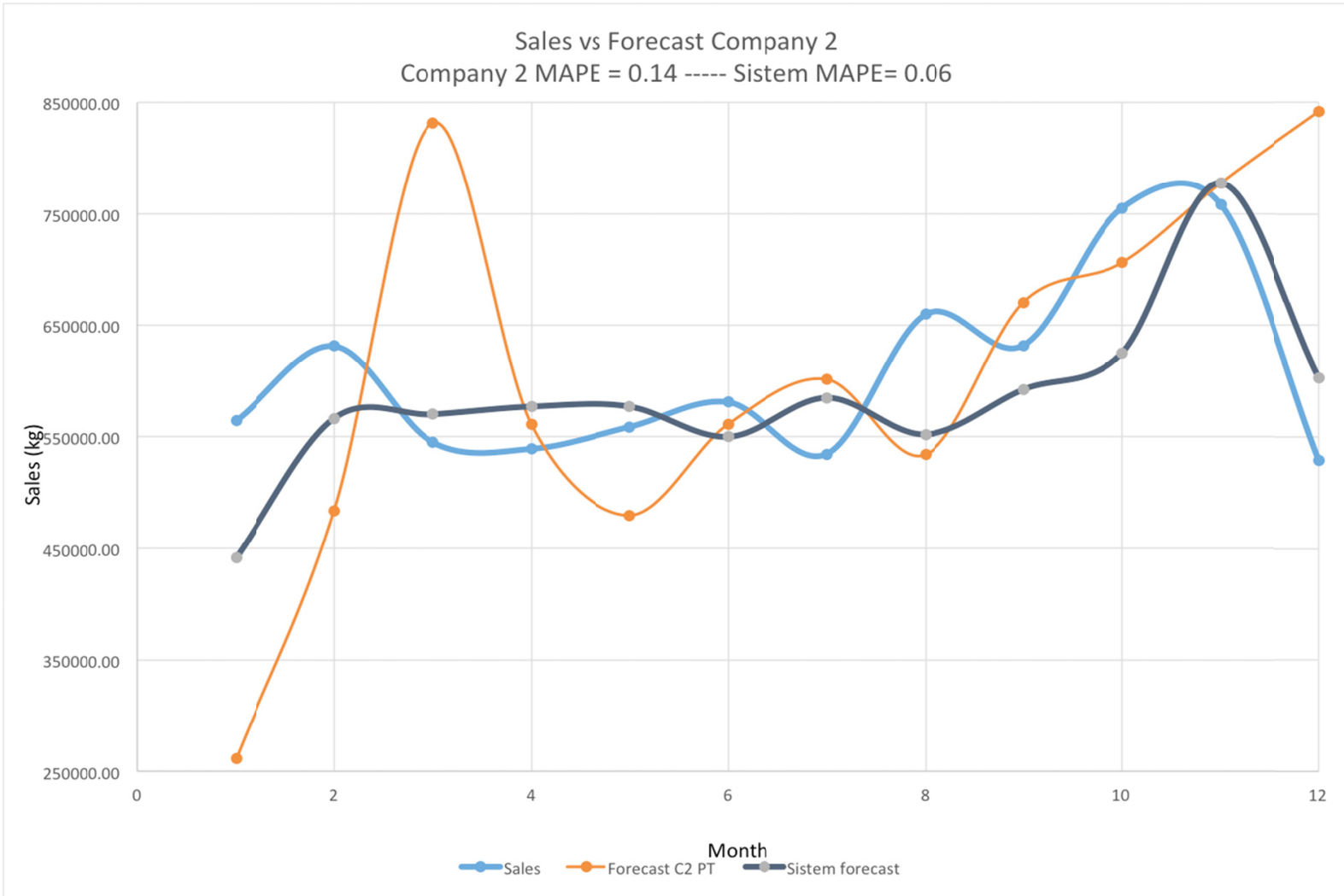

Figure 4. Comparison of the actual sales (Light blue line), with the sales forecast obtained by the trend projection technique in Company 2 (Orange line), and the values obtained by the present forecast system (Dark blue), with a MAPE $<<0.5$ 


\section{Application of Results}

The system developed can be applied to other companies in the same business by just changing the system matrix size in accordance to the number of products and months to be considered for the minor error technique in the sales forecasting system.

\section{Conclusions and Future Research}

The graphs of sales forecasts show different patterns for each material (trend, seasonal, cyclical, random, etc.); so it is necessary that in each case the most appropriate method of forecasting is determined. This approach is consistent with the findings of Perez, et al. (2012). It is required to feed the system with real monthly data for using in the developed computer program.

The best technique is the one with the lowest MAPE error value and it should be between 0 and 0.5 . For cases with MAPE error greater than 0.5 , the values obtained by the selected forecasting technique should be subjected to a correction by a qualitative technique.

In the present work, in $34 \%$ of the company 1 products and $29.2 \%$ of the company 2 products, a MAPE error less than 0.5 were obtained. This products or chemicals represent $51 \%$ and $89 \%$ of the respectively total sales for companies 1 and 2 .

The best prediction technique is different for each product. It was also determined that the best technique for a product varies with time according to market conditions, so it is necessary to be continually evaluating the best technique for each product at all times. We are currently working, on determining the number of periods to consider for each sales forecast in order to determine a significant MAPE value, and also in testing other error calculation methods to improve the forecasting system.

\section{References}

Anderson, D., Sweeney, D., William, T., \& Camm, J. (2011). Quantitative methods for business. Mexico, DF: Leaning CENGAGE.

Chase, B. R., \& Jacobs, F. R. (2014). Operations Management. Production and supply chain. Mexico, DF: Mc Graw Hill Education.

Chase, R., Aquilano, N., \& Jacobs, R. (2004). Production and Operations Management manufacturing and services. Mexico, DF: McGRAW-HILL.

Chopra, S., \& Meindl, P. (2008). Supply chain management. Mexico, DF: Prentice Hall.

Chu, C. H., \& Zhang, G. (2003). A comparative study of linear and nonlinear models for aggregate retail sales forecasting. International Journal of Production Economics, 86, 217-231.

Collier, D., \& Evans, J. (2009). Operations management services and value chain. Mexico, DF: CENGAGE Learning.

Corres, G., Esteban, A., García, J. C., \& Zarate, C. (2009). Time Series Analysis forecasts definition. Industrial Engineering Magazine, 8(1), 21-33.

Durbin, J., \& Koopman, S. (2001). Times Series Analysis by space methods. London: Oxford University Press.

Everet, A., \& Ronald, J. E. (2000). Administration production and operations. Mexico, DF: Prentice Hall Hispano American.

Heizer, J., \& Render, B. (2009). Principles of Operations Management. Mexico, DF: Pearson Prentice Hall.

Henke, J., \& Reitch, A. (2001). Business forecasting. New Jersey: Prentice-Hall.

Hill, C., \& Jones, G. (2009). Strategic Management. Mexico, DF: Mc.Graw Hill.

Monks, J. (2004). Operations Management. Mexico, DF: McGRAW-HILL.

Perez, R. A., Mosquera, S. A., \& Bravo, J. J. (2012). Application of Forecasting Models in consumer products. Biotechnology in the Agricultural Sector and Agroindustrial, 10(2), 117-125.

Taha, H. (2004). Operations Research. Mexico, DF: Pearson Education.

Thompsom, E. (2014). Striking a Banlance between Sales and Operations in the Forecasting Process. Journal of Business Forecasting, Winter, 2013-2014.

Vidal, C., Condoned, J., \& Contreras. (2004, September). Application of inventory models in a supply chain consumer products with a Bogeda and N outlets. Retrieved April 3, 2012, from http://web.ebscohost.com/ehost/pdfviewer/pdfviewer?vid=3\&hid=19\&sid=dd78005c-4cac-4fec-935b-a02f8fff8 $32 \mathrm{a} \% 40$ sessionmgr 10

Winston, W. (2005). Operations Research Applications and Algorithms. Mexico, DF: THOMSON. 